\title{
Possible origin of the slow-diffusion region around Geminga
}

\author{
Kun Fang ${ }^{1 \star}$ Xiao-Jun Bi ${ }^{1,2} \dagger$ Peng-Fei Yin ${ }^{1} \ddagger$ \\ ${ }^{1}$ Key Laboratory of Particle Astrophysics, Institute of High Energy Physics, Chinese Academy of Sciences, Beijing 100049, China \\ 2 School of Physical Sciences, University of Chinese Academy of Sciences, Beijing 100049, China
}

\begin{abstract}
Geminga pulsar is surrounded by a multi-TeV $\gamma$-ray halo radiated by the high energy electrons and positrons accelerated by the central pulsar wind nebula (PWN). The angular profile of the $\gamma$-ray emission reported by HAWC indicates an anomalously slow diffusion for the cosmic-ray electrons and positrons in the halo region around Geminga. In the paper we study the possible mechanism for the origin of the slow diffusion. At first, we consider the self-generated Alfvén waves due to the streaming instability of the electrons and positrons released by Geminga. However, even considering a very optimistic scenario for the wave growth, we find this mechanism DOES NOT work to account for the extremely slow diffusion at the present day if taking the proper motion of Geminga pulsar into account. The reason is straightforward as the PWN is too weak to generate enough high energy electrons and positrons to stimulate strong turbulence at the late time. We then propose an assumption that the strong turbulence is generated by the shock wave of the parent supernova remnant (SNR) of Geminga. Geminga may still be inside the SNR, and we find that the SNR can provide enough energy to generate the slow-diffusion circumstance. The TeV halos around PSR B0656+14, Vela X, and PSR J1826-1334 may also be explained under this assumption.
\end{abstract}

Key words: cosmic rays - ISM: individual objects: Geminga nebula - ISM: supernova remnants - turbulence

\section{INTRODUCTION}

The well-known $\gamma$-ray pulsar Geminga is surrounded by a multi-TeV $\gamma$-ray halo, which was first detected by Milagro (Abdo et al. 2007). In late 2017, the High-Altitude Water Cherenkov Observatory (HAWC) collaboration further reported the spatially resolved observation of the $\gamma$-ray halo (Abeysekara et al. 2017a). As these very-high-energy (VHE) $\gamma$ rays are emitted by electrons and positrons ${ }^{1}$ mainly through inverse Compton scattering of the cosmic microwave background photons, the surface brightness profile of the $\gamma$ ray emission can be a good indicator for the propagation of the electrons near the source. However, the derived diffusion coefficient of $\sim 60 \mathrm{TeV}^{2}$ electrons is hundreds times slower

\footnotetext{
* fangkun@ihep.ac.cn

† bixj@ihep.ac.cn

† yinpf@ihep.ac.cn

1 Electrons will denote both electrons and positrons hereafter.

2 The average energy of the $\gamma$ rays observed by HAWC is $20 \mathrm{TeV}$.

Considering both the inverse Compton scattering process and the power-law injection spectrum of the parent electrons, the average energy of the parent electrons for the $20 \mathrm{TeV} \gamma$ rays is $\sim 60$
}

than the average value in the Galaxy as inferred from the boron-to-carbon ratio (B/C) measurements (Aguilar et al. 2016). This is an evidence that the diffusion coefficient may be highly inhomogeneous in small scale. Investigating the origin of this slow-diffusion region could be meaningful to understand the particle propagation near cosmic-ray sources.

A plausible explanation is that the relatively large particle density near the source may lead to the resonant growth of Alfvén waves, which in turn scatter the particles and therefore suppress the diffusion velocity (Ptuskin et al. 2008; Malkov et al. 2013; D'Angelo et al. 2016). Based on this mechanism, the diffusion coefficient around Geminga can be significantly reduced in the case of a hard injection spectrum of electrons and a weak ambient magnetic field (this calculation is presented in Appendix A. See also Evoli et al. (2018)). However, the precondition of this interpretation is that Geminga need to be at rest so that the plenty of elec-

$\mathrm{TeV}$. We adopt the identical parameters with the original paper of HAWC to get this value, including the interstellar radiation field and the injection spectral index. 
trons produced in the early age of Geminga could create a slow-diffusion environment. While due to the proper motion of Geminga, it has already left $70 \mathrm{pc}$ away from its birthplace (Faherty et al. 2007), which means the observed slow-diffusion region must not be formed in the early age of Geminga. The injection power of Geminga in the present day should be much weaker than that in the early time, and we will show that the diffusion coefficient cannot be remarkably suppressed even in the abscence of wave dissipation.

Apart from the self-generated scenario, the slowdiffusion region around Geminga could also be a preexisting structure. The diffusion coefficient inside a supernova remnant (SNR) should be significantly smaller than that of the interstellar medium (ISM), as this region has been swept by the blast wave and acquired more turbulent energy. So if Geminga is still inside its associated SNR, it could be embedded in a region with small diffusion coefficient, which may explain the observed $\gamma$-ray halo. As Geminga may have a $70 \mathrm{pc}$ offset from its birthplace at the present day, there are works considering that Geminga has already left behind its associated SNR. However, we will show below that if the progenitor of Geminga is in a rarefied circumstance, the present scale of the SNR could be large enough to include Geminga inside. In the light of this explanation, the problems encountered in the self-confinement scenario could be avoided.

In this work, we first test the self-confinement picture in Section 2 with very optimistic assumptions, including the disregard of the wave dissipation. We consider the impacts brought by the proper motion of Geminga, which is an unavoidable factor. Then in Section 3, we introduce in detail the new interpretation of the inefficient diffusion halo, in which the electrons injected by Geminga are diffusing in the turbulent environment inside its parent SNR. In Section 4, we give some further discussion about this topic, including a brief analysis of some other $\mathrm{TeV}$ inefficient diffusion halos, and an alternative scenario of the preexisting kind of origin for the Geminga halo. Finally, we conclude in Section 5.

\section{THE SELF-CONFINED DIFFUSION SCENARIO}

A large density gradient of cosmic-ray particles can induce the streaming instability, which may amplify the Alfvén waves in background plasma (Skilling 1971). To derive the diffusion coefficient in the vicinity of a source, we must simultaneously solve the equations of particle transportation and the evolution of Alfvén waves. A full numerical solution of the coupled equations is presented in Appendix A. Here we only show an optimistic scenario for the turbulence growth where the energy loss of electrons and the Alfvén wave dissipation are ignored. The analysis shows clearly why the self-generated mechanism cannot work to stimulate the required turbulence.

We neglect the radiative energy loss of electrons, which leads to a larger gradient of the number density of electrons. Then the propagation equation is expressed as

$$
\frac{\partial N}{\partial t}-\nabla \cdot(D \nabla N)=Q
$$

where $N$ is the differential number density of electrons, $D$ is the diffusion coefficient, and $Q$ is the source function.

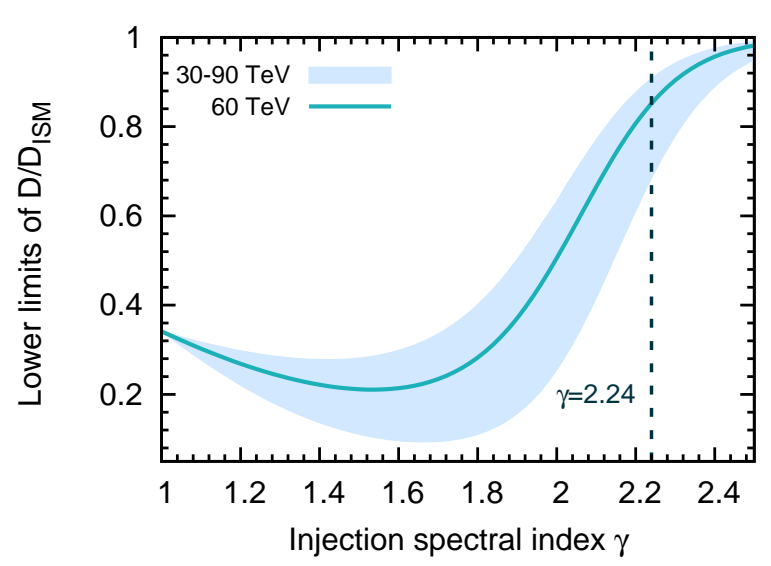

Figure 1. The lower limit of the diffusion coefficient around Geminga under the self-confinement scenario. The solid line is the case of $60 \mathrm{TeV}$, which is the mean energy of the parent electrons of the $\gamma$ rays observed by HAWC. The band shows the lower limit of diffusion coefficient varying from 30 to $90 \mathrm{TeV}$, corresponding to the energy range of HAWC observation. The dotted line marks the injection spectral index provided by HAWC.

The energy density of Alfvén waves is denoted with $W$, which is defined by $\int W(k) d k=\delta B^{2} / B_{0}^{2}$, where $k$ is the wave number, $B_{0}$ is the mean magnetic field strength, and $\delta B$ is the turbulent magnetic field. Here we ignore the wave dissipation and only consider the growth of the Alfvén waves through streaming instability. The evolution of $W$ can be then calculated by

$$
\frac{\partial W}{\partial t}=\Gamma_{\mathrm{cr}} W=-\frac{4 \pi v_{A} E_{\mathrm{res}}^{2}}{3 B_{0}^{2} k} \nabla N\left(E_{\mathrm{res}}\right)
$$

where $\Gamma_{\mathrm{cr}}=-4 \pi v_{A} E_{\mathrm{res}}^{2} /\left(3 B_{0}^{2} k W\right) \nabla N\left(E_{\mathrm{res}}\right)$ is the growth rate according to Skilling (1971), $v_{A}$ is the Alfvén speed, and $E_{\mathrm{res}}$ is the energy of electrons satisfying $r_{g}\left(E_{\mathrm{res}}\right)=1 / k$, where $r_{g}$ is the Larmor radius of electrons. Evoli et al. (2018) have proved that the expression of $\Gamma_{\mathrm{cr}}$ is also applicable for the streaming of electron-positron pairs. The diffusion coefficient is related with $W$ by (Skilling 1971)

$$
D\left(E_{\mathrm{res}}\right)=\frac{1}{3} r_{g} c \cdot \frac{1}{k W(k)} \text {. }
$$

Combining Equation (2) and (3), we get

$$
\frac{1}{D^{2}} \frac{\partial D}{\partial t}=\frac{4 \pi e v_{A} E}{B_{0} c} \nabla N .
$$

If Geminga is initially in a typical environment of ISM with $\delta B \ll B_{0}$, the diffusion coefficient along the magnetic field lines should be about $\left(\delta B / B_{0}\right)^{-4}$ times larger than the crossfield diffusion coefficient (Drury 1983). So the propagation of electrons should be initially in a tube of regular magnetic field lines, which corresponds to a one-dimensional diffusion. We set $x$ as the coordinate along the regular magnetic field lines, and we get the following expression from Equation (1) and (4):

$$
\frac{\partial}{\partial t}\left(N-\frac{B_{0} c}{4 \pi e v_{A} E} \frac{\partial \ln D}{\partial x}\right)=\delta(x) \dot{Q}(t),
$$

where we assume Geminga is a point-like source, and $\dot{Q}(t)$ is the time profile of electron injection. Then for any $x>0$, 
it can be derived from Equation (5) that

$$
N-\frac{B_{0} c}{4 \pi e v_{A} E} \frac{\partial \ln D}{\partial x}=0 .
$$

Integrating Equation (6) from $x$ to $\infty$, we finally obtain

$$
D(x)=D_{\mathrm{ISM}} \exp \left(-\frac{4 \pi e v_{A} E}{B_{0} c} \int_{x}^{\infty} N d x^{\prime}\right),
$$

with $D_{\text {ISM }}=D(\infty)$.

Geminga has a proper motion of about $200 \mathrm{~km}$ $\mathrm{s}^{-1}$ (Faherty et al. 2007), and the direction of motion is suggested to be nearly transverse to the line of sight (Caraveo et al. 2003). These indicate that Geminga has left its birthplace for about 70 pc now. Meanwhile, the motion of Geminga is almost perpendicular to the Galactic disk (Gehrels \& Chen 1993). This means that Geminga has been cutting the magnetic field lines of ISM, as the magnetic field in the Galactic disk is dominated by the horizontal component (Han \& Qiao 1994). Thus, the electrons injected in the early age of Geminga should have escaped along the magnetic field lines which is almost perpendicular to the path of Geminga motion and cannot help to generate the present slow-diffusion region. In other words, the slow-diffusion region around Geminga observed today must be formed in the recent age if the region is self-excited by Geminga.

We assume the electrons injected during the last third of the age of Geminga (228 kyr $342 \mathrm{kyr}$ ) contribute to the generation of the current slow-diffusion region; this should also be an optimistic assumption considering the very fast energy loss of high energy electrons. The injection time function is set to have the same profile with the spin-down luminosity of pulsar, which leads to $\dot{Q}(t, E)=\dot{Q}_{0}\left(1+t / \tau_{0}\right)^{-2} E^{-\gamma}$, where $\tau_{0}=10 \mathrm{kyr}$ (Hooper et al. 2017). We assume all the spindown energy of Geminga pulsar is converted to the injected electrons with energy from $1 \mathrm{GeV}$ to $500 \mathrm{TeV}$, to determine the normalization $\dot{Q}_{0}$. As we have neglected the energy loss of electrons, the following relation can be obtained according to particle conservation:

$$
2 S \int_{x}^{\infty} N\left(x^{\prime}, E\right) d x^{\prime}<\int_{t_{1}}^{t_{2}} \dot{Q}\left(t^{\prime}, E\right) d t^{\prime}
$$

where $t_{1}=228 \mathrm{kyr}, t_{2}=342 \mathrm{kyr}$, and $S$ is the cross-section of the magnetic flux tube which is assumed to have a scale of 1 pc. Combining Equation (7) and (8), we can then calculate the lower limit of the diffusion coefficient.

The Alfvén speed is decided by $B_{0}$ and the ion density $\rho_{i}$ as $v_{A}=B_{0} / \sqrt{4 \pi \rho_{i}}$. Then Equation (7) indicates that $D(x)$ is independent of $B_{0}$ in our calculation. Considering the morphology of the bow-shock structure observed in $\mathrm{X}$ ray (Caraveo et al. 2003) and the latest distance measure of Geminga (Faherty et al. 2007), the ISM density around Geminga $\rho_{\text {ISM }}$ is derived to be 0.02 atoms $\mathrm{cm}^{-3}$. Since the ionization around Geminga is very high (Caraveo et al. 2003), we have $\rho_{i} \approx \rho_{\text {ISM }}$. The injection spectral index $\gamma$ cannot be well constrained, as HAWC provides only the energy-integrated result at present.

In Figure 1, we present the lower limit of diffusion coefficient for varying $\gamma$. When $\gamma \approx 2.24$ as provided by HAWC, we have $D(60 \mathrm{TeV})>0.85 D_{\mathrm{ISM}}(60 \mathrm{TeV})$. For $60 \mathrm{TeV}$ particles, the minimum of the lower limit appears at $\gamma=1.54$, where $D(60 \mathrm{TeV})>0.21 D_{\text {ISM }}(60 \mathrm{TeV})$. However, this is still too far from the level of suppression required by HAWC observation, which is only about $10^{-3}$ of the normal diffusion

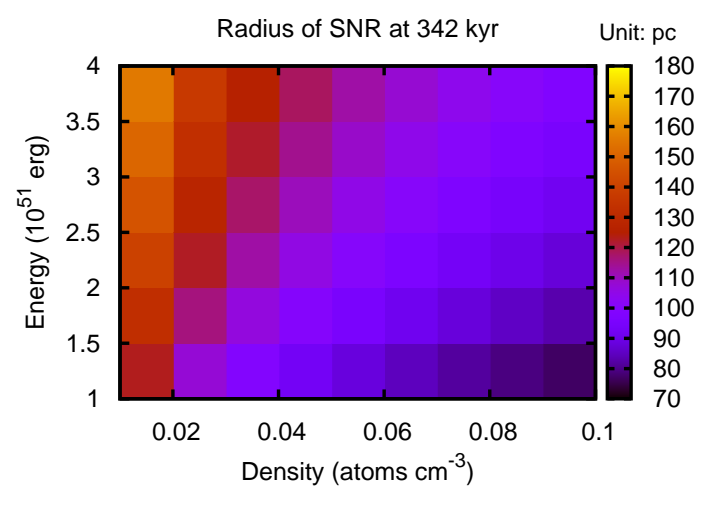

Figure 2. The radius of an SNR at the age of 342 kyr, with varying explosion energy and ambient mass density. The calculator provided by Leahy \& Williams (2017) is applied to get this figure.

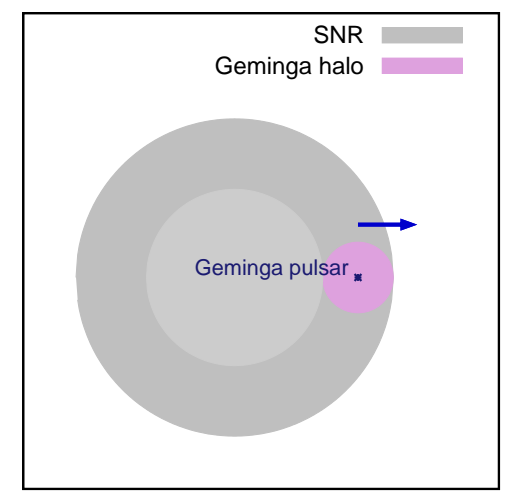

Figure 3. The sketch of the scenario introduced in Section 3. The arrow denotes the direction of the proper motion of Geminga pulsar. In the calculation of Section 3, the magnetic field turbulence is assumed to be distributed in the dark-gray shell.

value in ISM as determined by fitting the latest $\mathrm{B} / \mathrm{C}$ value in Yuan et al. (2017). Therefore it is clearly shown that the self-confinement mechanism cannot serve as the main reason for the suppression of $D(\sim 60 \mathrm{TeV})$ around Geminga.

\section{ELECTRON AND POSITRON DIFFUSION INSIDE THE SNR}

In the SNR shock frame, the upstream plasma loses part of kinetic energy when streaming through the shock front, and this part of energy is transfered into turbulence and thermal energy behind the shock (Bell 1978). Thus, the downstream region can be highly turbulent (Giacalone \& Jokipii 2007), although the turbulence will be gradually declined with the long-time evolution of SNRs. So if Geminga is still inside its associated SNR, the slow-diffusion region around it may be explained.

We first give an estimate of the possible scale of the Geminga SNR. We adopt the calculator provided by Leahy \& Williams (2017). This implement is created for 

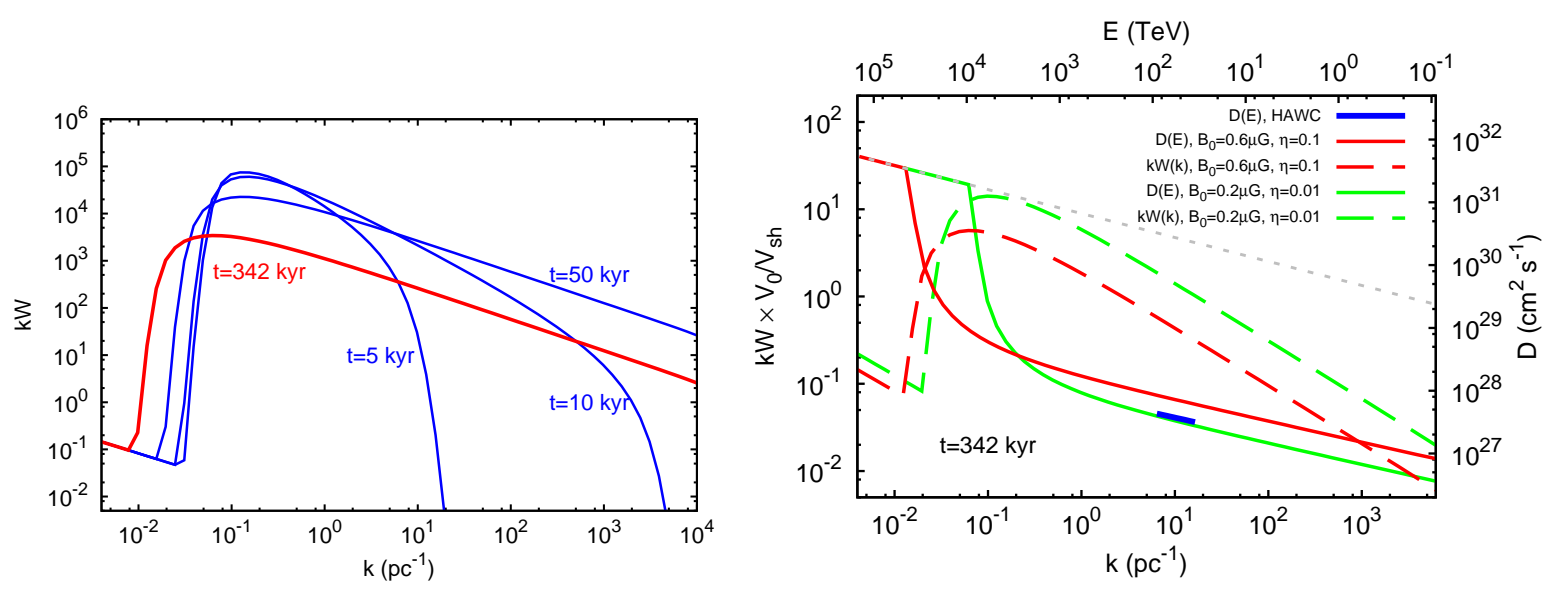

Figure 4. Left: the time evolution of the wave spectrum of the MHD turbulence, which is dominated by the wave cascading. Note that in this graph $W$ is not diluted with the expansion of the SNR. Right: the present-day turbulence spectrum inside the Geminga SNR and the corresponding diffusion coefficient, compared with the diffusion coefficient observed by HAWC. The gray dotted line is the diffusion coefficient in the ISM. Two different parameter sets are adopted: $B_{0}=0.6 \mu \mathrm{G}, \eta=10 \%$ (red); $B_{0}=0.2 \mu \mathrm{G}, \eta=1 \%$ (green).

modeling the evolution of SNRs, and consistently combines different models for different stages of SNR evolution. The SNR dynamic evolution is decided by the parameters such as the initial energy of the ejecta $E_{0}$ and the density of the ISM $n_{\text {ISM }}$. Figure 2 shows the radius of an SNR at the age of Geminga (342 kyr), with different $E_{0}$ and $n_{\text {ISM }}$. The ejecta mass is fixed at $1.4 M_{\odot}$. For a typical initial energy of $1 \times 10^{51} \mathrm{erg}$, the scale of the SNR can reach $\sim 100 \mathrm{pc}$ if the ambient density is relatively low with $n_{\text {ISM }}<0.05$ $\mathrm{cm}^{-3}$. We note that Geminga is in the southeast of Monogem Ring on the sky map (in the Galactic coordinate), and the distance of Monogem Ring is believed to be similar with that of Geminga. The ISM density in the south of Monogem Ring is derived to be $0.034 \mathrm{~cm}^{-3}$ (Knies et al. 2018), so we assume the same ambient density for the parent SNR of Geminga. In this case, the current scale of the SNR is 90 pc, if $E_{0}=10^{51} \mathrm{erg}$.

As mentioned above, Geminga has left its birthplace for about $70 \mathrm{pc}$ now. Considering the current radius of the Geminga SNR and the size of the observed Geminga halo ( $20 \mathrm{pc}$ ), we may envisage a scenario in which Geminga has been chasing the SNR shock, as presented in Figure 3. Meanwhile, the corresponding shock temperature is about $10^{5} \mathrm{~K}$ according to the calculator of Leahy \& Williams (2017), and the temperature inside the SNR should be higher. This is consistent with the high ionization degree around the pulsar wind nebula (PWN) of Geminga, as indicated by the measurement of the $\mathrm{H} \alpha$ luminosity (Caraveo et al. 2003).

The turbulent energy should be mainly generated in the very early age of an SNR, as the shock speed rapidly decreases after the ejecta dominated stage. For our parameter setting, the transition age of Geminga SNR from the ejecta dominated stage to the Sedov-Taylor stage is $\sim 850$ yr, which is negligible compared with the current age of Geminga. So it is reasonable to consider a burst-like injection for the turbulent energy. For simplicity, we assume that the turbulent energy is injected homogeneously into the SNR. Then we write the evolution equation of the magnetic field turbulence as

$$
\left\{\begin{array}{l}
\frac{\partial W}{\partial t}=\frac{\partial}{\partial k}\left(D_{k k} \frac{\partial W}{\partial k}\right) \\
W(0, k)=Q_{W} \delta(t) \delta\left(k-k_{0}\right)+W_{\mathrm{ISM}}
\end{array}\right.
$$

where we assume the evolution is dominated by the turbulent cascading, with the Kolmogorov type diffusion coefficient $D_{k k}=0.052 v_{A} k^{7 / 2} W^{1 / 2} \quad$ (Miller \& Roberts 1995). The wave damping due to the ion-neutral interaction is not significant for a high ionization environment (Kulsrud \& Cesarsky 1971). The initial condition is the sum of the injection term and the ISM term, and the latter is related to $D_{\text {ISM }}$ by Equation (3). We assume the injection scale of the MHD turbulence to be $l_{0}=10 \mathrm{pc}$, corresponding to $k_{0}=0.1 \mathrm{pc}^{-1}$; the size of Geminga can indeed reach $\sim 10 \mathrm{pc}$ in the very early age for our case. The normalization of the injection term is expressed by

$$
Q_{W}=\frac{\eta E_{0} / V_{0}}{B_{0}^{2} / 8 \pi}
$$

where $\eta$ is the conversion efficiency of the magnetic field turbulence, and $V_{0}=4 \pi l_{0}^{3} / 3$. López-Coto \& Giacinti (2018) argued that the HAWC observation of Geminga halo favors an rms magnetic field of $3 \mu \mathrm{G}$. So if the outer scale of the Kolmogorov type turbulence is $10 \mathrm{pc}$, the mean field $B_{0}$ should be about $0.6 \mu \mathrm{G}$.

We numerically solve Equation (9) with the finite difference method. As Equation (9) is a non-linear problem, we adopt the predictor-corrector method to linearize the difference equation set. The wave number $k$ spans a broad range of magnitude in the numerical calculation, so we convert $k$ into the logarithmic scale with $x=\log _{10}\left[k\left(\mathrm{~cm}^{-1}\right)\right]$. The step lengths are set to be $\Delta x=0.1$ and $\Delta t=2$ yr to ensure the accuracy of the solution.

The evolution of the wave spectrum is presented in the left of Figure 4 . The wave spectrum expands in the $k$ space and converges to the Kolmogorov type $\left(W \sim k^{-5 / 3}\right)$ after the age of $\sim 10 \mathrm{kyr}$. Then the intensity of the spectrum gradually decreases until the present day. However, with the expansion of the SNR, the turbulent energy injected in the 
early should be diluted. To compare with the observation, we assume that the turbulent energy is now homogeneously distributed in a shell 50-90 pc from the SNR center, which includes the Geminga halo in. For an old SNR, the mass is indeed distributed in the outer part (Cioffi et al. 1988), and so does the turbulence. Then the turbulent energy density is diluted from $W$ to $W \times V_{0} / V_{\mathrm{sh}}$, and $V_{\mathrm{sh}}$ is the volume of the shell where the turbulence is mainly distributed. Note that this is a conservative estimation for $W$. In the real case, $W$ is gradually diluted with the expansion of the SNR, not instantaneously at the present day. This implies that $D_{k k}$ should be smaller compared with the calculation above. As shown in the left of Figure 4, the wave spectrum has been decreasing after tens of kyr. A smaller $D_{k k}$ will lead to a higher current wave spectrum.

The current diffusion coefficient corresponding to $W \times$ $V_{0} / V_{\mathrm{sh}}$ is shown in the right of Figure 4 . For the case of $B_{0}=0.6 \mu \mathrm{G}, D(E)$ can be close to the value reported by HAWC with $\eta=10 \%$, which means the SNR is energetic enough to explain the inefficient diffusion environment of Geminga. Recently, Liu et al. (2019a) analyze the X-ray data of XMM-Newton and Chandra around the Geminga pulsar and derive an upper limit of the diffuse X-ray flux, which corresponds to a maximum magnetic field of $1 \mu \mathrm{G}$ within $\sim 1 \mathrm{pc}$ around the Geminga pulsar. If this conclusion can be extrapolated to a larger neighbourhood of Geminga, we may have a maximum $B_{0}$ of $0.2 \mu \mathrm{G}$. Then in the right of Figure 4 , we also show the case of $B_{0}=0.2 \mu \mathrm{G}$. In this case, the theoretical $D(E)$ can accommodate the observed value only with a conversion efficiency of $1 \%$ for the magnetic field turbulence. The reason is that $Q_{W}$ can be larger for a smaller $B_{0}$. Moreover, the cascading time scale is approximately $\tau_{c} \sim k_{0}^{2} / D_{k k} \sim k_{0}^{-3 / 2}\left(\eta E_{S N}\right)^{-1 / 2} V_{0}^{1 / 2} \rho_{i}^{1 / 2}$. The cascading time scale is larger for a smaller $\eta$, which results in a stronger current wave spectrum for a fixed $Q_{W}$, as explained in the last paragraph. Thus to explain the observed $D(E)$, the required conversion efficiency $\eta$ is positively correlated with $B_{0}$.

\section{DISCUSSION}

\subsection{Other TeV halos}

The mechanism to explain the slow diffusion around Geminga proposed in the previous section can be examined in other similar $\mathrm{TeV} \gamma$-ray halos around pulsars. Besides Geminga there are other pulsars that are observed to be surrounded by slow diffusion halos in TeV. The spatial profile of $\gamma$-ray emission around PSR B0656+14 was reported by HAWC along with that of Geminga, and the indicated diffusion coefficient is 5 times larger compared with the Geminga case (Abeysekara et al. 2017a). While unlike Geminga, the associated SNR of PSR B0656+14, namely the Monogem Ring, is still observable in X-ray (Bunner et al. 1971; Plucinsky et al. 1996), as it is much younger than Geminga ( 100 kyr). The position of PSR B0656+14 on the sky map is well inside the Monogem Ring, while the observation of the PWN of PSR B0656+14 suggests that the motion of the pulsar is almost parallel to the line of sight (Bîrzan et al. 2016). Since Monogem Ring is an extended structure with a scale of $\sim 80$ pc (Knies et al. 2018), the
$\mathrm{TeV}$ halo of PSR B0656+14 can still be included by the SNR as long as its radial velocity is not faster than $600 \mathrm{~km}$ $\mathrm{s}^{-1}$. If so, the origin of the slow-diffusion region could also be explained by the scenario of Section 3.

Vela X, as the PWN of Vela pulsar, is close to the center of Vela SNR (Sushch et al. 2011). H.E.S.S has detected an extended $\mathrm{TeV}$ structure around Vela pulsar with a scale of $\sim 6$ pc (Aharonian et al. 2006a; Abramowski et al. 2012), which is considered to be correlated with the X-ray filament (Hinton et al. 2011). The TeV halo is more extended than the X-ray filament, and the derived magnetic field is only $\sim 4 \mu \mathrm{G}$, much smaller than that close to the pulsar (Hinton et al. 2011). So it is possible that the TeV structure is produced by the escaping electrons that are wandering in the turbulent environment inside the Vela SNR. Huang et al. (2018) also indicates that Vela X should be surrounded by a slow-diffusion environment, so that its lepton flux at the Earth will not conflict with the current experiments. Besides, the anomalously large extended $\mathrm{TeV}$ halo around PSR J1826-1334 (HESS J1825-137, Aharonian et al. 2006b; Khangulyan et al. 2018) could also be ascribed to the diffusion of electrons inside its host SNR.

On the other hand, we pay attention to another source PSR B1957+20, around which no TeV structure has been detected so far. PSR B1957+20 is an old millisecond pulsar, which is definitely traveling in the ISM now. The bow-shock PWN associated to the pulsar has been detected by Chan$d r a$ in $0.3-8 \mathrm{keV}$ (Stappers et al. 2003; Huang et al. 2012), and the magnetic field of PWN is estimated to be $17.7 \mu \mathrm{G}$ (Huang et al. 2012). So the parent electrons of the X-ray emission should be as high as tens of $\mathrm{TeV}$, which means this PWN can indeed accelerate electrons to VHE. Besides, Aharonian et al. (1997) pointed out that ground based telescopes should be able to detect VHE $\gamma$-ray emission around the pulsar if the spin-down luminosity of the pulsar is larger than $10^{34}(r / 1 \mathrm{kpc})^{2}$ erg s$~^{-1}$, where $r$ is the distance of the pulsar. For the case of PSR B1957+20, $r$ is inferred as 2.5 kpc (Cordes \& Lazio 2002), and the spin-down luminosity of the pulsar is $7.48 \times 10^{34} \mathrm{erg} \mathrm{s}^{-1}$ (Manchester et al. 2005), meeting the criterion above. All these imply that if the accelerated electrons are effectively confined near the source, $\mathrm{TeV}$ structure ought to be revealed. However, according to the present work, the VHE electrons may not be able to bound themselves by the self-generated waves, and the turbulence in the ISM is far from adequate to confine the escaping electrons, unlike the case inside the SNR. Thus, plenty of electrons might have effectively spread out and the nondetection of VHE emission can be understood.

\subsection{Is Geminga inside a stellar-wind bubble?}

The scenario described in Section 3 may not be the unique possible case for a preexisting slow-diffusion environment. It is also possible that Geminga is running into an unrelated turbulent region at present. Kim et al. (2007) discovered a large ring-like structure in $\mathrm{H} \alpha$ emission that is centered at $\left(97.14^{\circ}, 21.33^{\circ}\right)$ in equatorial coordinates, dubbed the 'Gemini H $\alpha$ Ring'. As can be seen in Figure 5, the most intense part of the Geminga $\mathrm{TeV}$ halo is included by the Gemini $\mathrm{H} \alpha$ Ring in projection. There is evidence that the Gemini $\mathrm{H} \alpha$ Ring is interacting with the Monogem Ring (Kim et al. 2007). As the distance of Monogem Ring is estimated to be 


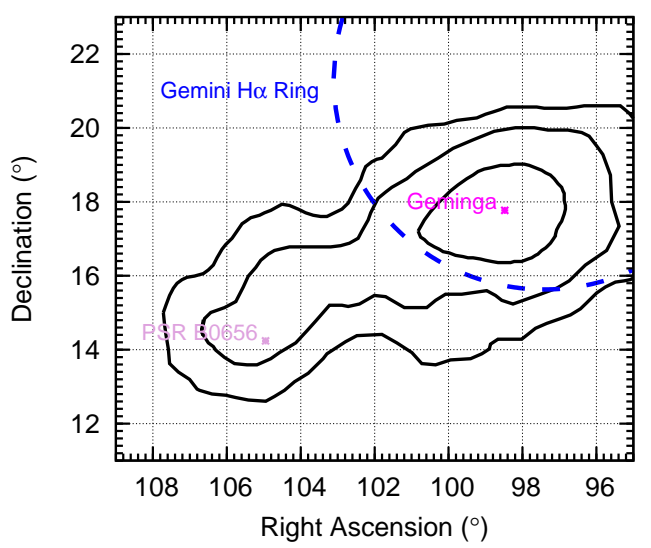

Figure 5. The positions of Geminga and the Gemini $\mathrm{H} \alpha \mathrm{Ring}$ on the sky map. The contours of the HAWC significance map between 1 and $50 \mathrm{TeV}(5 \sigma, 7 \sigma$, and $10 \sigma)$ are shown in black (Abeysekara et al. 2017a). The blue dashed line represents the Gemini H $\alpha$ Ring (Knies et al. 2018).

$\sim 300$ pc (Knies et al. 2018), the Gemini H $\alpha$ Ring should have a similar distance. Knies et al. (2018) pointed out that the Gemini $\mathrm{H} \alpha$ Ring is most likely a stellar-wind bubble, as there are several $\mathrm{OB}$ type stars in the direction of the $\mathrm{H} \alpha$ ring with distances of 200-350 pc. Meanwhile, the uncertainty of the trigonometric parallax of Geminga is still large, and the derived distance ranges from $190 \mathrm{pc}$ to $370 \mathrm{pc}$ (Faherty et al. 2007). Thus, Geminga is possibly inside the stellar-wind bubble now, and the shocked wind may provide Geminga a turbulent circumstance. Besides, the high ionization and low density environment of Geminga are also consistent with the features of a stellar-wind bubble (Castor et al. 1975).

\section{CONCLUSION}

We study the possible origin of the slow-diffusion region around Geminga observed by HAWC. Considering the proper motion of Geminga, we verify that the mechanism of self-generated Alfvén waves due to the streaming instability cannot work to produce such a low diffusion coefficient even in the most optimistic scenario where the energy loss of electrons and the dissipation of the Alfvén waves are neglected. The reason is simple as Geminga is too weak to generate enough high energy electrons at the late age. We get an analytical result of the lower limit of the diffusion coefficient, which are at most suppressed to about 0.2 times of the ISM value at $60 \mathrm{TeV}$. This is much larger than the value required by the HAWC observation, which derives a diffusion coefficient hundreds times smaller than that of the ISM.

We further propose a scenario that the slow diffusion is a preexisting effect, as Geminga may still be inside its unidentified parent SNR. We show that if the ambient density is low, the scale of the SNR can be large enough to include Geminga and the $\mathrm{TeV}$ halo inside. We assume that the magnetic field turbulence is injected inside the SNR in the very early age of the SNR, and then the evolution of the turbulence is dominated by the wave cascading with the
Kolmogorov form. Our calculation indicates that the diffusion coefficient observed by HAWC can be reproduced if $1-10 \%$ of the initial energy of the SNR is converted into the magnetic field turbulence, considering the uncertainty of the magnetic field strength. Thus, the SNR can provide enough energy to explain the slow-diffusion halo around Geminga. We also point out that our estimation should be a conservative one, which means the required conversion efficiency may be smaller. Another possible interpretation of the slow diffusion is also briefly presented, in which Geminga is now running into the stellar-wind bubble that creates the Gemini $\mathrm{H} \alpha$ Ring.

We further discuss some other sources with $\mathrm{TeV}$ halos, such as PSR B0656+14, Vela X, and PSR J1826-1334. These cases also favor our new interpretation. Recently, Liu et al. (2019b) propose an alternative scenario that the $\mathrm{TeV}$ halo of Geminga is not attributed to the strong turbulence, but interpreted by the anisotropy diffusion of the electrons along the local regular magnetic field which is presumed to be aligned with the line of sight towards Geminga. It is still ambiguous if the inefficient diffusion region around pulsar is universal or not. As the ground-based Cherenkov instruments have identified plenty of VHE $\gamma$-ray sources associated with pulsars (of course many of them are PWNe rather than halos produced by escaping electrons) (Abeysekara et al. 2017b; H. E. S. S. Collaboration et al. 2018), we expect to investigate more cases in the future work. Besides, if energyresolved observation of Geminga halo could be provided in the future, it is very helpful to give further judgment to the origin of the slow-diffusion region.

\section{ACKNOWLEDGEMENT}

We thank Prof. Hui Li and Dr. Yu-Dong Cui for helpful discussions. We also thank the anonymous reviewer for the constructive suggestions. This work is supported by the National Key Program for Research and Development (No. 2016YFA0400200) and by the National Natural Science Foundation of China under Grants No. U1738209, 11851303.

\section{REFERENCES}

Abdo, A. A., Allen, B., Berley, D., et al. 2007, ApJ, 664, L91

Abeysekara, A. U., Albert, A., Alfaro, R., et al. 2017a, Science, 358,911

-. 2017b, ApJ, 843, 40

Abramowski, A., Acero, F., Aharonian, F., et al. 2012, A\&A, 548, A38

Aguilar, M., Ali Cavasonza, L., Ambrosi, G., et al. 2016, Physical Review Letters, 117, 231102

Aharonian, F., Akhperjanian, A. G., Bazer-Bachi, A. R., et al. 2006a, A\&A, 448, L43

-. 2006b, A\&A, 460, 365

Aharonian, F. A., Atoyan, A. M., \& Kifune, T. 1997, MNRAS, 291,162

Bell, A. R. 1978, MNRAS, 182, 147

Bîrzan, L., Pavlov, G. G., \& Kargaltsev, O. 2016, ApJ, 817, 129

Bunner, A. N., Coleman, P. L., Kraushaar, W. L., \& McCammon, D. 1971, ApJ, 167, L3

Caraveo, P. A., Bignami, G. F., De Luca, A., et al. 2003, Science, 301, 1345

Castor, J., McCray, R., \& Weaver, R. 1975, ApJ, 200, L107 
Cioffi, D. F., McKee, C. F., \& Bertschinger, E. 1988, ApJ, 334, 252

Cordes, J. M., \& Lazio, T. J. W. 2002, ArXiv Astrophysics eprints, astro-ph/0207156

D'Angelo, M., Blasi, P., \& Amato, E. 2016, Phys. Rev. D, 94, 083003

Drury, L. O. 1983, Reports on Progress in Physics, 46, 973

Evoli, C., Linden, T., \& Morlino, G. 2018, Phys. Rev. D, 98, 063017

Faherty, J., Walter, F. M., \& Anderson, J. 2007, Ap\&SS, 308, 225

Fang, K., Bi, X.-J., Yin, P.-F., \& Yuan, Q. 2018, ApJ, 863, 30

Gehrels, N., \& Chen, W. 1993, Nature, 361, 706

Giacalone, J., \& Jokipii, J. R. 2007, ApJ, 663, L41

H. E. S. S. Collaboration, Abdalla, H., Abramowski, A., et al. 2018, A\&A, 612, A1

Han, J. L., \& Qiao, G. J. 1994, A\&A, 288, 759

Hinton, J. A., Funk, S., Parsons, R. D., \& Ohm, S. 2011, ApJ, $743, \mathrm{~L} 7$

Hooper, D., Cholis, I., Linden, T., \& Fang, K. 2017, Phys. Rev. D, 96, 103013

Huang, R. H. H., Kong, A. K. H., Takata, J., et al. 2012, ApJ, 760,92

Huang, Z.-Q., Fang, K., Liu, R.-Y., \& Wang, X.-Y. 2018, ApJ, 866,143

Khangulyan, D., Koldoba, A. V., Ustyugova, G. V., Bogovalov, S. V., \& Aharonian, F. 2018, ApJ, 860, 59

Kim, I.-J., Min, K.-W., Seon, K.-I., et al. 2007, ApJ, 665, L139

Knies, J. R., Sasaki, M., \& Plucinsky, P. P. 2018, MNRAS, 477, 4414

Kulsrud, R. M., \& Cesarsky, C. J. 1971, Astrophys. Lett., 8, 189

Leahy, D. A., \& Williams, J. E. 2017, AJ, 153, 239

Liu, R.-Y., Ge, C., Sun, X.-N., \& Wang, X.-Y. 2019a, ApJ, 875, 149

Liu, R.-Y., Yan, H., \& Zhang, H. 2019b, arXiv e-prints, arXiv:1904.11536

López-Coto, R., \& Giacinti, G. 2018, MNRAS, 479, 4526

Malkov, M. A., Diamond, P. H., Sagdeev, R. Z., Aharonian, F. A., \& Moskalenko, I. V. 2013, ApJ, 768, 73

Manchester, R. N., Hobbs, G. B., Teoh, A., \& Hobbs, M. 2005, AJ, 129, 1993

Miller, J. A., \& Roberts, D. A. 1995, ApJ, 452, 912

Plucinsky, P. P., Snowden, S. L., Aschenbach, B., et al. 1996, ApJ, 463, 224

Ptuskin, V. S., Zirakashvili, V. N., \& Plesser, A. A. 2008, Advances in Space Research, 42, 486

Skilling, J. 1971, ApJ, 170, 265

Stappers, B. W., Gaensler, B. M., Kaspi, V. M., van der Klis, M., \& Lewin, W. H. G. 2003, Science, 299, astro-ph/0302588

Sushch, I., et al. 2011, A\&A, 525, A154

Yuan, Q., Lin, S.-J., Fang, K., \& Bi, X.-J. 2017, Phys. Rev. D, 95, 083007

\section{APPENDIX A: NUMERICAL SOLUTION OF THE SELF-CONFINEMENT SCENARIO}

Neglecting the proper motion of Geminga, we numerically solve the complete forms of Equation (1) and (2) in the following. The radiative cooling of electrons and the damping of Alfvén waves are considered, then Equation (1) and (2) are rewritten as

$$
\frac{\partial N}{\partial t}-\nabla \cdot(D \nabla N)-\frac{\partial}{\partial E}(b N)=Q
$$

and

$$
\frac{\partial W}{\partial t}+v_{A} \nabla W=\left(\Gamma_{\mathrm{cr}}-\Gamma_{\mathrm{dis}}\right) W
$$

The calculation of the cooling rate $b$ is identical with that in Fang et al. (2018), and the wave cascading is approximated by a damping term $\Gamma_{\text {dis }}=0.052 v_{A} k^{3 / 2} W^{1 / 2}$ for simplicity (Evoli et al. 2018). In fact, for the ISM that is not infulenced by the source, the wave growth and convection can be neglected, while the wave dissipation still exists. We universally add a compensatory growth term to keep intact the diffusion coefficient far from the source.

We solve Equation (A1) and (A2) iteratively in the onedimensional scenario. For Equation (A1), we apply the operator splitting method to deal with the diffusion operator and the energy-loss operator seperately. For each operator, we derive the differencing scheme with the finite volume method. This is important especially for the diffusion operator, as $D$ can be changed abruptly in space. One may refer to Fang et al. (2018) for the details of the differencing schemes. The intial value of $N$ is zero everywhere. For the boundary conditions, we set the the maximum injection energy to be $500 \mathrm{TeV}$. The typical scale of the Galactic random field is 100 pc, which means the one-dimensional diffusion is only valid within this scale around the source. If particles escape farther, the diffusion should switch to three-dimensional, and $N$ will sharply declines. So we set the spacial outer boundary at $100 \mathrm{pc}$, namely $N(100 \mathrm{pc})=0$. The radius of the onedimensional flux tube is assumed to be $1 \mathrm{pc}$. As to Equation (A2), we discretize it with the well-known upwind scheme. The initial $W$ is decided by the diffusion coefficient in the ISM. To ensure the accuracy of the solutions, $D \Delta t /(\Delta x)^{2}$ and $v_{A} \Delta t / \Delta x$ should not be much larger than 1 , where $\Delta t$ is the time step and $\Delta x$ is the radial step for both Equation (A1) and (A2). As $D_{\mathrm{ISM}}(60 \mathrm{TeV}) \approx 3 \times 10^{30} \mathrm{~cm}^{2} \mathrm{~s}^{-1}$, we set $\Delta t=0.1 \mathrm{yr}$ and $\Delta x=1 \mathrm{pc}$.

The spectral index $\gamma$ of the injection spectrum and the mean magnetic field $B_{0}$ are important parameters for the self-confinement scenario. The former affects the growth rate of the Alfvén waves, while the later is decisive for the damping rate of the waves. In the left of Figure A1, we show the calculated $D(60 \mathrm{TeV})$ in the current age of Geminga with different $\gamma$ and $B_{0}$. For the case of harder $\gamma(1.5)$ and smaller $B_{0}(0.6 \mu \mathrm{G})$, the diffusion coefficient around Geminga is significantly suppressed even in the current age, and the average $D$ within $20 \mathrm{pc}$ is comparative to the result of HAWC. As can be seen in the right of Figure A1, the diffusion coefficient declines quickly in the early age, then the wave damping dominates and the diffusion coefficient gradually rises.

However, in addition to the unrealistic assumption that Geminga is at rest, the assumption of one-dimensional diffusion is not always valid. The turbulence need to be weak, namely $\delta B \ll B_{0}$. The right of Figure A1 shows that in the early age of Geminga, the diffusion coefficient can be suppressed to very low value, corresponding to strong turbulence. When $\delta B$ approaches $B_{0}$, the diffusion mode should switch to three-dimensional, and the wave growth due to streaming instability is significantly reduced compared with the case of one-dimensional. This implies that the diffusion coefficient cannot be reduced to so low as calculated here, while a self-consistent calculation should be complex and beyond the scope of this work. 

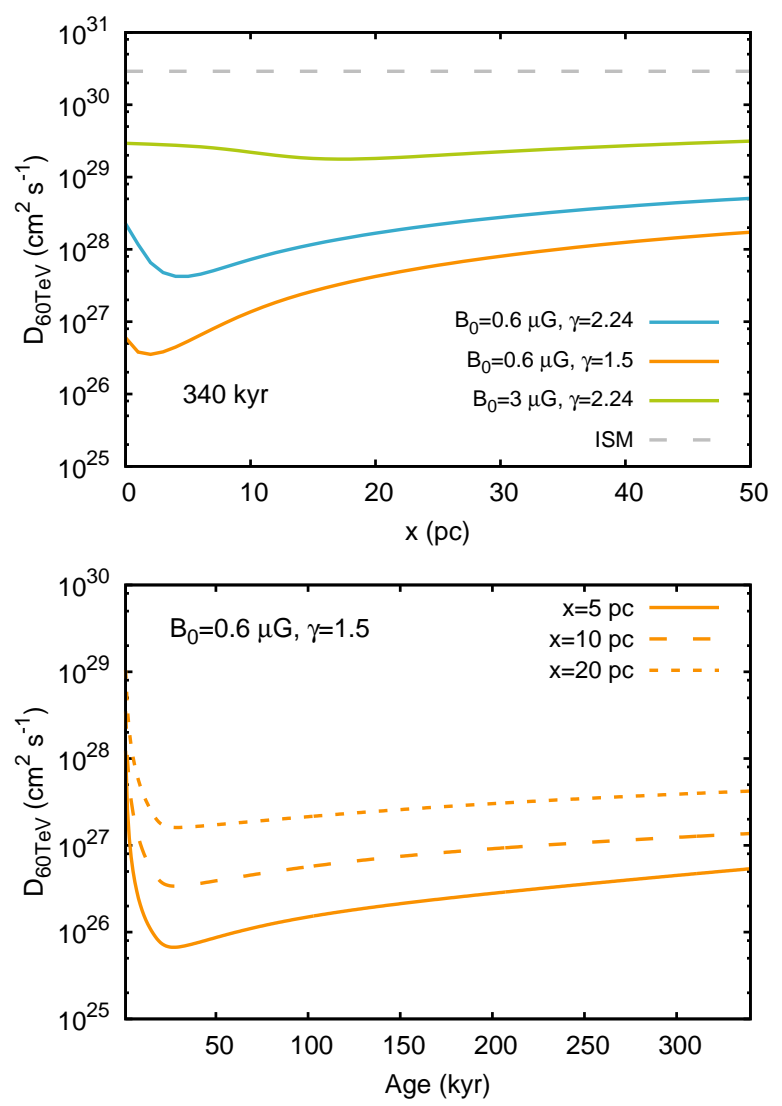

Figure A1. The result of the numerical solution to the selfconfined propagation of electrons, assuming Geminga is at rest. Top: $D(60 \mathrm{TeV})$ in the current age of Geminga, for different combinations of $B_{0}$ and $\gamma$. Bottom: the evolution of $D(60 \mathrm{TeV})$ at different distances from Geminga. 